\title{
A turbulence study in Dwarf Irregular galaxies
}

\author{
Margarita Valdez-Gutiérrez ${ }^{1}$ and Ivânio Puerari ${ }^{2}$ \\ ${ }^{1}$ Instituto de Astronomía - Universidad Nacional Autónoma de México, campus Ensenada, \\ Ensenada, B. C., Mexico \\ email: mago@astrosen.unam.mx \\ ${ }^{2}$ INAOE, Tonantzintla, Puebla, Mexico \\ email: puerari@inaoep.mx
}

\begin{abstract}
We present preliminary results of a Fourier transform power spectra analysis carried out on a sample of dwarf irregular galaxies and Local Group members. This project is intended to study turbulence and the ISM structure in irregulars as a class.
\end{abstract}

Keywords. Turbulence, ISM: structure, galaxies: dwarf, galaxies: irregular

\section{Results and Discussion}

Our galaxy sample is composed by six Local Group members observed in the $\mathrm{H} \alpha$ line. Observations were carried out using the $2.1 \mathrm{~m}$ telescope at the Observatorio Astronómico Nacional at San Pedro Mártir B. C. México. The data acquisition was performed by means of the Fabry-Perot interferometer PUMA (Rosado et al. 1995) in direct imaging mode and covering a field of view of $10^{\prime}$. The Fourier transform power spectra were calculated from cuts along the galaxies major axes and tested to reproduce a noise power spectra. Power spectra slope values for four of the galaxies of our sample range from 1.3 to 1.5. This is in agreement with Willett et al. (2005) results for irregular galaxies with high star formation rates. On the contrary, for WLM and IC1613, their slopes are almost zero corresponding to systems with low star formation rate per unit area. These preliminary results suggest some dependence on the star formation rate. Our findings test our reduction programs and methodology with previous results reported by Willett al. (2005). The analysis of individual emission regions in the galaxies of the sample is in progress (Valdez-Gutiérrez et al. 2007, in preparation).

\section{References}

Willett, K.W, Elmegreen, B.G. \& Hunter D.A. 2005, AJ 129, 2186

Rosado, M. et al. 1995, Rev. Mexicana Astron. Astrofis. Ser. Conf. 3, 263 